\title{
Complexity in supramolecular analogues of frustrated magnets at high pressure
}

$\underline{\text { Andrew Brian Cairns }}^{1}$, Matthew J. Cliffe ${ }^{2}$, Joseph A. M. Paddison ${ }^{3}$, Dominik Daisenberger ${ }^{4}$, Matthew G. Tucker ${ }^{5}$, François-Xavier Coudert $^{6}$, Andrew L. Goodwin ${ }^{7}$, Mohamed Mezouar ${ }^{1}$

${ }^{1}$ ESRF - The European Synchrotron, Grenoble, France, ${ }^{2}$ Department of Chemistry, University of Cambridge, Cambridge, United Kingdom, ${ }^{3}$ Department of Physics, University of Cambridge, Cambridge, United Kingdom, ${ }^{4}$ Diamond Light Source, Didcot, United Kingdom, ${ }^{5}$ Spallation Neutron Source, Oak Ridge, United States, ${ }^{6}$ CNRS \& Chimie ParisTech, Paris, France, 7 Inorganic Chemistry Laboratory, Department of Chemistry, University of Oxford, Oxford, United Kingdom

E-mail: andrew.cairns@esrf.fr

Frustrated magnets-materials where magnetic interactions compete with each other or with the underlying lattice geometry -are of great interest to physicists, chemists and crystallographers alike [1]. Particularly intriguing are the complex and unexpected collective states that emerge due to frustration, such as magnetic vortices and magnetic monopoles. This "emergent" behaviour is also found in the conceptually-related field of systems chemistry, where collective states arise on a fundamentally different lengthscale to that of the interactions themselves [2]. The famous 'spin ices' highlight this mapping: the low-temperature magnetic state of $\mathrm{Ho}_{2} \mathrm{Ti}_{2} \mathrm{O} 7$ has tetrahedral spin clusters that adopt a two-in-two-out arrangement that is directly related to the orientation of water molecules in cubic ice [1]. A major challenge is that many of the most exotic frustrated magnets predicted are not physically realisable. We have shown how chemically 'simple' metal cyanides can act as supramolecular analogues of two-dimensional triangular XY magnets [3]. The key structural degrees of freedom-the relative shifts of neighbouring gold(I)/silver(I) cyanide chains in $\mathrm{AgCN}, \mathrm{AuCN}$ and $\mathrm{AgAu}(\mathrm{CN})_{2}-$ are mathematically equivalent of the phase angle of rotating planar spins. So complex states predicted for this family of magnets are realised in the structural chemistry of these cyanide polymers for the first time.

Undoubtedly the most exciting avenue of research is the exploration of excitations and/or response of these materials to external stimuli. Are the screw dislocations observed in $\operatorname{AgAu}(\mathrm{CN})_{2}$ fixed or mobile? Do we observe pinning/unpinning phenomena known for spin vortices? Are high-pressure structures dominated by volume or interaction effects (or a combination of both)? Here we present studies on the high-pressure behaviour of $\mathrm{AgCN}$ and $\mathrm{AgAu}(\mathrm{CN}) 2, \mathrm{using}$ a combination of synchrotron powder X-ray diffraction, Monte Carlo simulations, and quantum mechanical calculations. A series of phase transitions for each is interpreted primarily in the context of our theoretical understanding of frustrated magnets, but may also give insight into the behaviour of complex magnetic states and materials design under extreme conditions. The critical importance of metallophilic interactions on the structures and properties of these materials is highlighted.

[1] Harris, M. J. et al. (1997). Phys. Rev. Lett., 79, 2554-2557.

[2] Nitschke, J. R. (2009). Nature, 462, 736-738.

[3] Cairns, A. B. et al. (2016). Nature Chem., 8, 442-447.

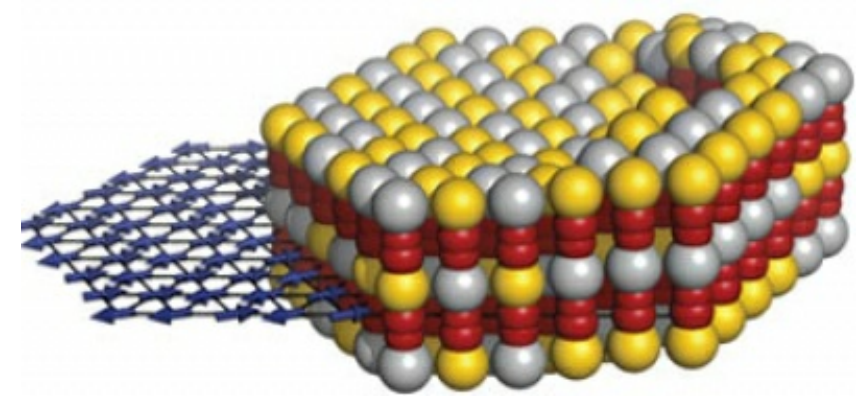

Keywords: frustrated magnets, structural analogues, high pressure 